\title{
Involvement of Chemokines and a CD4-Positive T Cell Subset in the Development of Conjunctival Secondary Lymphoid Follicles in an Atopic Keratoconjunctivitis Mouse Model
}

\author{
Jun Shoji ${ }^{\mathrm{a}}$ Yoko Nakanishi ${ }^{\mathrm{b}}$ Akiko Ishimori ${ }^{\mathrm{a}}$ Tohru Sakimoto $^{\mathrm{a}}$ Noriko Inada ${ }^{\mathrm{a}}$ \\ Norimichi Nemoto ${ }^{b}$ \\ ${ }^{a}$ Division of Ophthalmology, Department of Visual Sciences and ${ }^{b}$ Division of Human Pathology, Department of \\ Pathology and Microbiology, Nihon University School of Medicine, Tokyo, Japan
}

\section{Key Words}

Atopic keratoconjunctivitis · Chemokine $\cdot$ CD20 · iCALT .

$\mathrm{NC} / \mathrm{Nga}$ mouse

\begin{abstract}
Background: Massive B cell lymphoid hyperplasia and its associated factors may play a role in exacerbating inflammation in allergic disorders. We here investigated the chemokines and CD4-positive T cell subset involved in the development of secondary lymphoid follicles (iCALT) in conjunctival tissues in an atopic keratoconjunctivitis mouse model (AKC mouse). Methods: NC/Nga mice were divided into three groups: AKC (percutaneous sensitization and instillation of crude house dust mite antigen), AD (percutaneous sensitization only) and $C$ (untreated control). Pathological changes in the conjunctival tissues of each group were investigated using histological and immunohistochemical detection of CD4 and CD20. Furthermore, tissue sections of iCALT (AKC-iCALT subgroup) and conjunctiva without iCALT (AKC-conjunctiva subgroup) were obtained from AKC mice using laser-assisted microdissection. mRNA expression of chemokine and T cell subset-related transcription factors were compared between the AKC-iCALT and AKC-conjunctiva subgroups using polymerase chain reaction (PCR) array and real-time reverse transcription-PCR (RT-PCR) methods. Results: iCALT with
\end{abstract}

central aggregation of CD20-positive B cells and CD4-positive $T$ cell infiltration surrounding $B$ cells was observed in the palpebral conjunctival tissue of the AKC group, but not in that of the $A D$ and $C$ groups. Chemokine and T cell subsetrelated transcription factor expression was confirmed using real-time RT-PCR, with significant increases in $\mathrm{Cc} / 5, \mathrm{Cc} / 17$, CxI20,Cxcl3, Ccr7, Foxp3 and T-bet mRNA expression in the AKC-iCALT subgroup compared with those in the AKC-conjunctiva subgroup. Conclusions: We concluded that CCL5, CCL17 and CCL20, as well as T-bet- and Foxp3-positive lymphocytes may be iCALT-related factors and that iCALT-related chemokines are worth evaluating as biomarkers.

๑) 2015 S. Karger AG, Basel

\section{Introduction}

Atopic keratoconjunctivitis (AKC) and vernal keratoconjunctivitis (VKC) are severe and chronic allergic conjunctival diseases (ACD) that result from immediate hypersensitivity. However, AKC and VKC have a complicated pathophysiology, including proliferative conjunctival lesions that cannot be explained by the immediate hypersensitivity theory. Besides the proliferative conjunctival lesions (e.g. giant papillae and limbal gelatinous infiltration), the clinical characteristics of patients with AKC or

\section{KARGER 125}

(c) 2015 S. Karger AG, Base

$1018-2438 / 15 / 1673-0147 \$ 39.50 / 0$

E-Mail karger@karger.com

www.karger.com/iaa
Correspondence to: Dr. Noriko Inada

Division of Ophthalmology, Department of Visual Sciences

Nihon University School of Medicine

30-1 Oyaguchi-Kamicho, Itabashi-ku, Tokyo 173-8610 (Japan)

E-Mail inada.noriko@ nihon-u.ac.jp 
VKC include high serum levels of antigen-specific IgE antibodies, increased expression and secretion of inflammatory cytokines (IL-4, IL-5), soluble cytokine receptors (soluble IL-6 receptor), and chemokines (eotaxin, IL-8) in tears [1-6]. In particular, high levels of these cytokines and chemokines in the tears of patients with severe ACD are thought to originate from the conjunctival epithelium and lacrimal gland, and the mast cells, eosinophils and type 2 helper T (Th2) cells in conjunctival tissue, and play a critical role in the pathogenesis of allergic inflammation.

In severe collagen disease and allergic disorders, B cell lymphoid hyperplasia is observed in target organs, such as the synovium in rheumatoid arthritis, or the lungs in asthma. Therefore, invasion by B cells may be a crucial factor in the pathogenesis of severe allergic disorders. In the bronchus and lung tissues of patients with asthma, $\mathrm{B}$ cells, in addition to eosinophils and T cells, are associated with allergic inflammation. Additionally, inducible bronchus-associated lymphoid tissue (iBALT) is an ectopic secondary lymphoid tissue that forms in the lung after pulmonary inflammation or infection $[7,8]$. iBALT has histological characteristics similar to Peyer's patches and acts as a conventional lymphoid tissue [9].

There are many similarities between airway inflammation in asthma and conjunctival inflammation in $\mathrm{AKC} /$ VKC. For instance, in a histological study of giant papillae in patients with VKC, some demonstrated small B lymphoid follicle formations $[10,11]$. These histological findings suggested the possibility that inducible conjunctivaassociated lymphoid tissue (which we henceforth term iCALT) forms due to the severe allergic inflammation present in patients with AKC/VKC. CALT is extranodal lymphoid tissue that belongs to mucosa-associated lymphid tissue (MALT), which is similar to Peyer's patches and BALT [12-16]. MALT, including CALT and BALT, is extranodal lymphoid tissue that is involved in antibody production and in the induction of inducible regulatory $\mathrm{T}$ cells that occur in the mucosal immune system $[17,18]$. Therefore, ectopic lymphoid tissues, such as iCALT, may be involved in the immunological pathophysiology of allergic inflammation in the conjunctiva. However, the pathophysiology underlying the development of iCALT in the conjunctival tissues of patients with ACD is poorly understood.

NC/Nga mice develop atopic dermatitis (AD)-like skin lesions spontaneously under conventional conditions, but do not do so under specific pathogen-free (SPF) conditions. However, it is difficult to set up an experimental environment in which NC/Nga mice persistently develop AD-like skin lesions. Therefore, attempts have recently been made to establish an NC/Nga mouse model that is antigen sensitized under SPF conditions [19]. Yamamoto et al. [20] reported that repeated application with Dermatophagoides farinae extract ointment, together with barrier disruption, could induce AD-like skin lesions in NC/Nga mice under SPF conditions. Furthermore, we have been able to produce an AKC mouse model based on NC/Nga mice with AD-like skin lesions, in which severe allergic conjunctivitis is developed after instillation of an ophthalmic ointment that contains crude $D$. farinae antigen [21]. The AKC mouse model is characterized by high anti-Df-specific IgE antibodies in serum and by AKC-compatible conjunctivitis, including pathological changes of eosinophilic inflammation with massive lymphoid hyperplasia, pseudotubular formations in the conjunctival epithelium, and granuloma formation in the limbal conjunctiva [22]. However, the inflammatory reactions in the AKC mouse conjunctiva are not as severe when considering the clinical and pathological findings in human AKC patients [21].

There is a need for biomarkers that can be used in a clinical test in order to select therapeutic drugs that are appropriate to the varied pathogeneses of AKC/VKC. Therefore, we were prompted to investigate iCALT-related factors, over and above the Th2-related factors that are associated with allergic inflammation. The purpose of this study was thus to investigate the histopathological characteristics of iCALT in conjunctival tissues and to identify iCALT-related chemokine and transcriptional factors in the CD4-positive T cell subset in conjunctival tissues that can be considered as biomarkers for iCALT, using the AKC mouse model.

\section{Materials and Methods}

\section{Animals}

The animal experiments in this study were approved by the Institutional Animal Care and Use Committee of Nihon University School of Medicine. All animals were treated in accordance with the Association for Research in Vision and Ophthalmology (ARVO) resolutions regarding animals used in research.

Eight-week-old female NC/Nga mice, reared under SPF conditions, were purchased from Charles River Japan (Yokohama, Japan). Food and tap water were provided freely.

\section{AKC Mouse Model}

AKC-like ocular surface lesions were induced in mice (AKC mice) according to a previously described method [21]. Either dermatologic ointment containing house dust mite (HDM; D. $f a$ rinae) body (Dfb; Biostir ${ }^{\circledR} \mathrm{AD}$, Biostir; Kobe, Japan) or Dfb ophthalmic ointment, containing $5 \mathrm{mg} \mathrm{Dfb} / \mathrm{g}$ white petrolatum (PROPETO $^{\circledR}$, Maruishi Pharmaceutical, Tokyo, Japan), was used. 
Fig. 1. Experimental protocol. NC/Nga mice were divided into three groups: AKC $(\mathrm{n}=8), \mathrm{AD}(\mathrm{n}=8)$ and $\mathrm{C}($ control; $\mathrm{n}=8)$ groups, depending on treatment (1) and treatment (2). Treatment (1): percutaneous sensitization using HDM crude antigencontaining ointment. Treatment (2): instillation of HDM crude antigen-containing ophthalmic ointment. Histological examination was performed on the three groups. Specimens from each group were divided into the conjunctiva subgroups (AKC-conjunctiva, $\mathrm{AD}$-conjunctiva and $\mathrm{C}$-conjunctiva) and the iCALT subgroup (AKCiCALT) using LAMD. Each sample was evaluated using RT-PCR; the results from AKC-conjunctiva and AKC-iCALT were compared using PCR array.

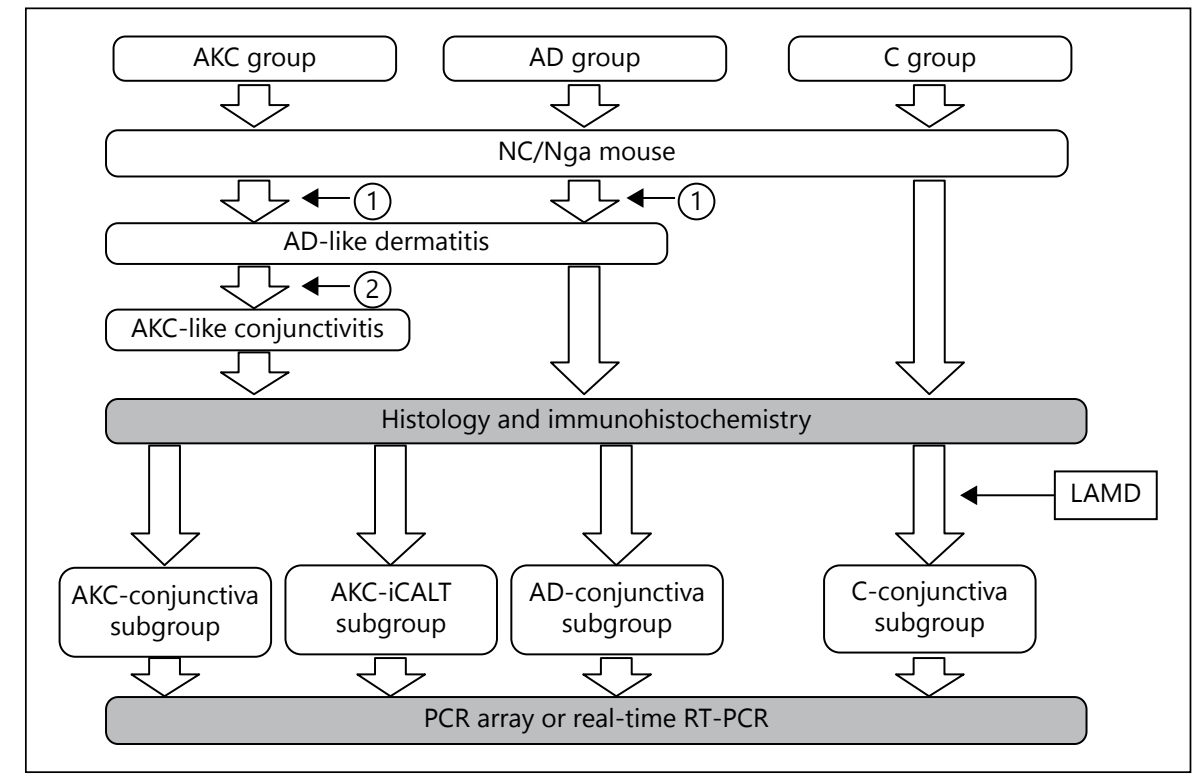

$\mathrm{NC} / \mathrm{Nga}$ mice were sensitized by repeated topical applications of the Dfb dermatologic ointment (percutaneous sensitization), and challenged by instillation of Dfb ophthalmic ointment (ophthalmic challenge). On the first sensitization (day 1), we applied $100 \mathrm{mg}$ of Dfb dermatologic ointment onto the denuded dorsal skin and on the surface of both ears of the mice. This topical application was repeated twice a week. By day 17, AD-like skin lesions developed. On days 18-20, an ophthalmic challenge was performed once a day by instillation of $5 \mathrm{mg}$ of Dfb ophthalmic ointment in the AKC group ( $n=8$; fig. 1$)$. The $\operatorname{AD}$ group $(n=8)$ received percutaneous sensitization without any ophthalmic instillation (fig. 1 ). The control group ( $\mathrm{C}$ group; $\mathrm{n}=8$ ) received neither percutaneous sensitization nor ophthalmic instillation. At $24 \mathrm{~h}$ after the final instillation, after the mice had been euthanized with an overdose of pentobarbital, the eyeballs and eyelids were removed in all groups.

\section{Histology and Immunofluorescence}

Histological Examination. Entire specimens of the conjunctiva and cornea of mice were embedded in paraffin after fixation with a $4 \%$ paraformaldehyde solution (WAKO, Tokyo, Japan). Sections ( $5 \mu \mathrm{m}$ thick) were stained with hematoxylin and eosin (HE) for standard histology, or with direct fast scarlet (DFS) for the detection of eosinophils.

Immunohistochemistry. For immunohistochemical analysis, the paraffin-embedded sections were incubated in 5\% normal rabbit serum (Vector Laboratories, Burlingame, Calif., USA) for $30 \mathrm{~min}$ to block nonspecific reactions. Then, slides were incubated with the primary anti-mouse CD20 goat polyclonal antibody (clone: M-20; Santa Cruz Biotechnology, Dallas, Tex., USA) for $60 \mathrm{~min}$ at room temperature. Detection was achieved using the universal immunoenzyme polymer method with a Histofine ${ }^{\circledR}$ mouse MAX PO kit (Nichirei Biosciences, Tokyo, Japan), according to the manufacturer's recommendations. The specimens were then placed in a solution of 3,3'-diaminobentidine tetra-hydrochloride $(20 \mathrm{mg} / 100$ $\mathrm{ml}$ ) dissolved in $0.05 \mathrm{M}$ Tris- $\mathrm{HCl}$ buffer ( $\mathrm{pH} 7.6$ ) containing $0.005 \%$
$\mathrm{H}_{2} \mathrm{O}_{2}$ for $5 \mathrm{~min}$. Finally, the slides were counterstained with methyl-green and were examined by light microscopy (BH-2, Olympus, Tokyo, Japan). For immunofluorescence analysis, entire conjunctiva and cornea specimens were embedded in an optimal cutting temperature compound (OCT; Sakura Seiki, Tokyo, Japan) and frozen over dry ice-isopentane. Frozen sections ( $7 \mu \mathrm{m}$ thick) were fixed in $100 \%$ methanol (WAKO) for $1 \mathrm{~min}$. For double-immunofluorescence staining, slides were blocked for $30 \mathrm{~min}$ at room temperature with $5 \%$ normal donkey serum (Vector Laboratories) in phosphate-buffered saline. After blockade, the slides were incubated overnight at $4{ }^{\circ} \mathrm{C}$ with an anti-mouse CD4 rat monoclonal antibody (clone: GK1.5; Abcam, Tokyo, Japan), which was detected with Alexa Fluor ${ }^{\circledR} 488$-labeled donkey anti-rat IgG (Life Technologies, Tokyo, Japan). Next, slides were incubated for $90 \mathrm{~min}$ at $30^{\circ} \mathrm{C}$ with an anti-mouse CD20 goat polyclonal antibody (clone: $\mathrm{M}-20$; Santa Cruz Biotechnology), which was detected with Alexa Fluor ${ }^{\circledR}$ 647-labeled donkey anti-goat IgG (Life Technologies). Finally, the slides were counterstained with DAPI (4'6-diaminido2-phenylindole)-Fluoromount-G (SouthernBiotech, Birmingham, Ala., USA). Images were recorded with a BIOREVO digital camera (BZ-9000; Keyence Japan, Osaka, Japan).

\section{Polymerase Chain Reaction}

Laser-Assisted Microdissection. Conjunctival tissues from mice in the AKC, $\mathrm{AD}$ and $\mathrm{C}$ groups were embedded in OCT compound (Sakura Seiki, Tokyo, Japan) without prior fixation. Frozen blocks of conjunctival tissues were sectioned ( $20 \mu \mathrm{m}$ thick) onto RNAse-free polyethylene naphthalate-coated glass slides (Leica Microsystems, Tokyo, Japan). The tissue sections were subsequently exposed to a 30 -second incubation in cold $100 \%$ methanol, followed by immersion in $\mathrm{dH}_{2} \mathrm{O}$ and $0.02 \%$ toluidine blue, and air-dried. Laser-assisted microdissection (LAMD) was immediately performed using the LMD7000 system (Leica Microsystems, Wetzlar, Germany). The conjunctiva from the AKC, $\mathrm{AD}$ and $\mathrm{C}$ groups (AKC-conjunctiva, $\mathrm{AD}$-conjunctiva and $\mathrm{C}$ conjunctiva subgroups, respectively), consisting of conjunctival 

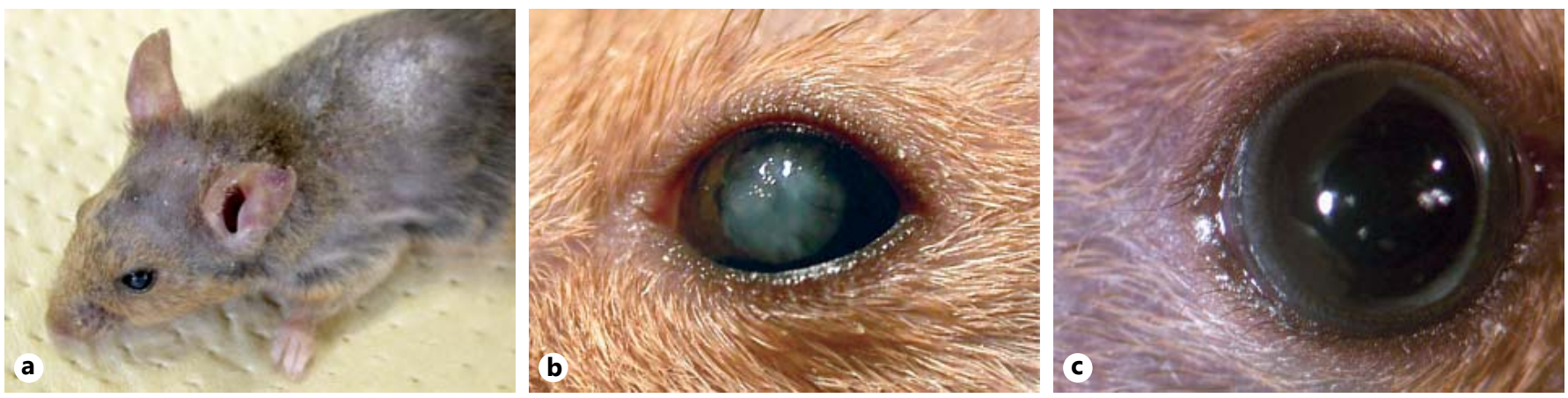

Fig. 2. Clinical observations in the AKC mouse model. The photographs show an NC/Nga mouse that had developed $\mathrm{AD}$-like dermatitis (a), and severe conjunctivitis and cataracts (b). In contrast, lid swelling and the conjunctival hyperemia were not observed in the NC/Nga mice of group C (c).

tissues without iCALT, and the iCALT from the AKC group (AKC-iCALT subgroup), consisting of conjunctival tissues with massive infiltration of lymphocytes into subepithelial tissues, was classified according to the LAMD of the sections. The dissected samples were collected into Thermo-Tube caps (Thermo Scientific Japan, Yokohama, Japan) containing $10 \mu \mathrm{l}$ of mineral oil (Life Technologies). Total RNA was extracted as previously described [23] and the samples were stored at $-80^{\circ} \mathrm{C}$ until required for use.

PCR Array. To identify differences in chemokine-related gene expression between the AKC-iCALT $(n=1)$ and AKC-conjunctiva $(\mathrm{n}=1)$ subgroups, cDNA was synthesized with an $\mathrm{RT}^{2}$ First Strand Kit (Qiagen, Hilden, Germany) and samples were analyzed for the expression of 84 genes encoding chemokines or associated factors and receptors, using the $\mathrm{RT}^{2}$ Profiler PCR Chemokine array (PAMM-022ZA; Qiagen). Data were analyzed using $\mathrm{RT}^{2}$ Profiler PCR Array software and $\Delta \Delta \mathrm{C}_{\mathrm{t}}$-based fold changes were calculated.

Real-Time RT-PCR. For real-time reverse transcription polymerase chain reaction (real-time RT-PCR), RNA was extracted from specimens of the AKC-iCALT $(\mathrm{n}=8)$, AKC-conjunctiva $(\mathrm{n}=$ $8)$, AD-conjunctiva $(n=8)$, and C-conjunctiva subgroups $(n=8)$. cDNA was synthesized using the High Capacity cDNA Reverse Transcription Kit (Life Technologies Japan). To assess the expression of chemokines and their receptor mRNA in the conjunctiva subgroup among the $\mathrm{AKC}, \mathrm{AD}$ and $\mathrm{C}$ groups, real-time $\mathrm{RT}-\mathrm{PCR}$ was performed using TaqMan Gene Expression Assays (Life Technologies Japan) for mouse Ccl17/TARC (thymus and activationregulated chemokine; Mm00516136_m1), Ccl22/MDC (Mm00436439_m1) and Ccr4 (Mm00438271_m1). In addition, to compare the expression of chemokine and T cell subclass-related transcription factor mRNAs between the AKC-iCALT and AKCconjunctiva subgroups in the AKC group, real-time RT-PCR was again performed for mouse Ccl5/RANTES (regulated upon activation, normal $\mathrm{T}$ cell expression, and presumably secreted; Mm01302427_m1), Ccl17/TARC (Mm00516136_m1), Ccl20/ MIP-3a (macrophage inflammatory protein-3a; Mm01268754_ m1), Cxcl3 (Mm01701838_m1), Cxcl13 (Mm04214185_s1), Ccr7 (Mm00475162_m1), Tbx21/T-bet (Mm00450960_m1), Gata3 (Mm00484683_m1), Foxp3 (Mm00475162_m1) and Rorc/ROR $\gamma \mathrm{t}$ (Mm01261022_m1). The data were analyzed using the $\Delta \Delta \mathrm{C}_{\mathrm{t}}$ method.
Statistical Analysis

Real-time RT-PCR results were evaluated using the nonparametric Steel test, for investigation of differences among the AKC, $\mathrm{AD}$ and $\mathrm{C}$ groups, while the Mann-Whitney $\mathrm{U}$ test was used to analyze differences between the AKC-iCALT and AKC-conjunctiva subgroups. $p$ values $<0.05$ were considered to indicate statistical significance.

\section{Results}

\section{Ophthalmic Challenge in AD Mice Results in Severe}

Allergic Conjunctivitis

AKC (percutaneous sensitization and ophthalmic challenge by crude HDM antigen), AD (percutaneous sensitization only) and $\mathrm{C}$ mice (untreated control) were observed clinically. All of the mice in the AKC group developed $\mathrm{AD}$-like dermatitis (fig. 2a) and severe allergic conjunctivitis (fig. 2b), but not those in the $\mathrm{AD}$ and $\mathrm{C}$ groups (fig. 2c). In the $\mathrm{AKC}$ group, severe conjunctival hyperemia was seen in all mice and gelatinous conjunctival swelling at the conjunctival limbus was observed in 3 of 8 mice with severe conjunctivitis. Cataract (fig. 2b) was present in 1 of 8 mice in the AKC group.

\section{Histological and Immunohistochemical Observations Show Allergic Inflammation and $\mathrm{CALT}$ in \\ Subconjunctival Tissues of AKC Mice}

We first performed histological investigations to ascertain the presence of allergic inflammation in the conjunctiva and to search for CALT and iCALT. Following HE and DFS staining, no allergic inflammation was observed in the $\mathrm{AD}$ or the $\mathrm{C}$ group (fig. 3a, b). However, AKC sections displayed a large accumulation of inflammatory cells with eosinophil and lymphocyte infiltration in the subconjunctival tissues (fig. 3c). 

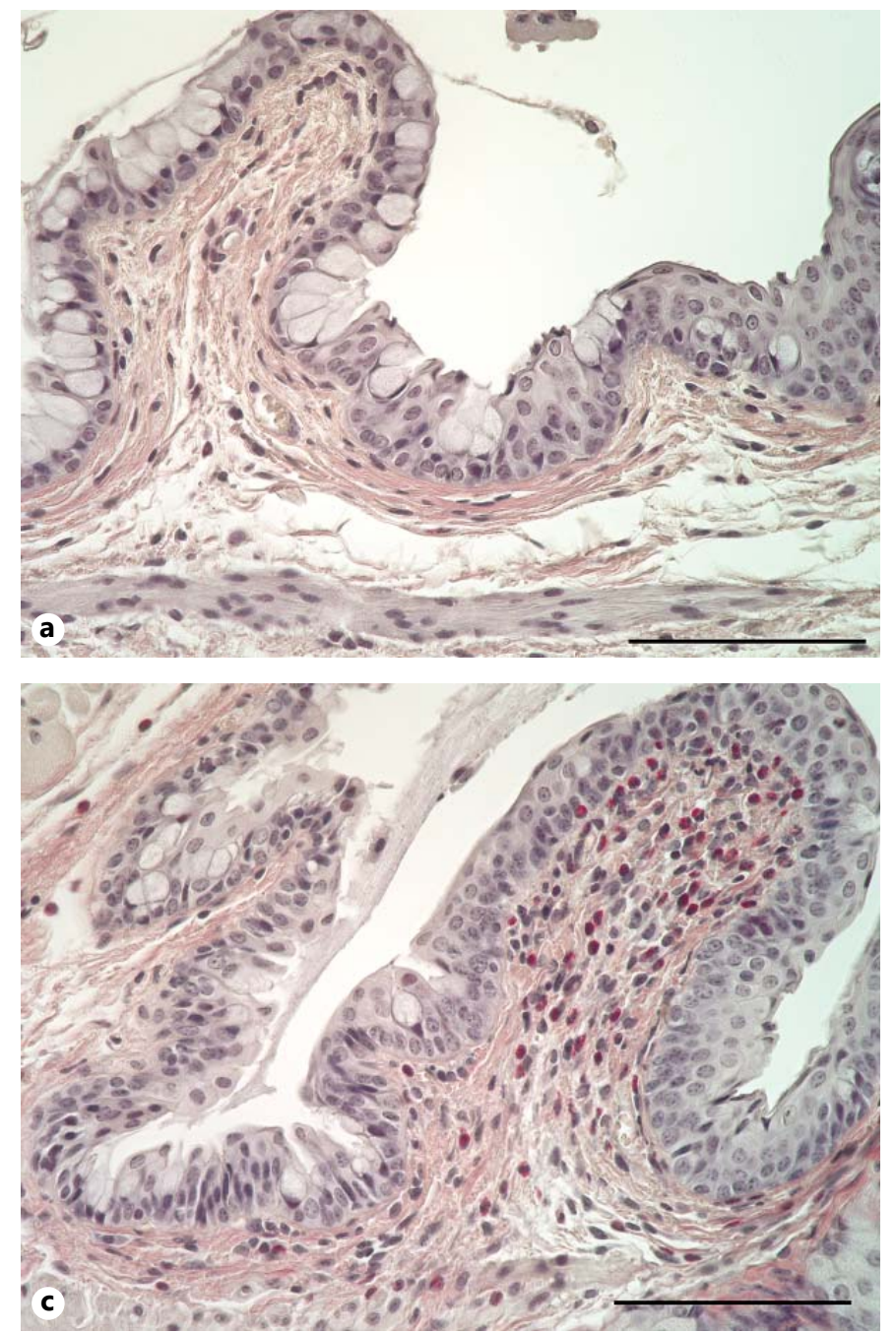

Fig. 3. Histological features of palpebral conjunctiva in the AKC, $\mathrm{AD}$ and $\mathrm{C}$ groups. Eosinophilic infiltration, detected by DFS staining, was absent in the $\mathrm{AD}$ group (a) and the $\mathrm{C}$ group (b). However, in subconjunctival tissues in the AKC group (c), allergic inflammation with eosinophils and lymphocytes infiltration was ob-

Moreover, in the mouse palpebral conjunctiva of the AKC group, follicular lymphoid hyperplasia, comprising CD20-positive cells, was observed in subconjunctival tissue (fig. 3d, $4 \mathrm{a}, \mathrm{b}$ ). In contrast, sections from the $\mathrm{AD}$ and $\mathrm{C}$ groups did not show any appreciable accumulation of lymphocytes or inflammatory cells, including eosinophils in the palpebral conjunctiva. In all groups, follicular lymphoid hyperplasia, comprising CD20-positive cells, was observed in the subepithelial tissue of the nictitating membrane (fig. 4c, d).

Immunofluorescence staining revealed CD20-positive $B$ cell aggregates in the central follicular lymphoid hyper-

Secondary Lymphoid Follicles in AKC

Mice
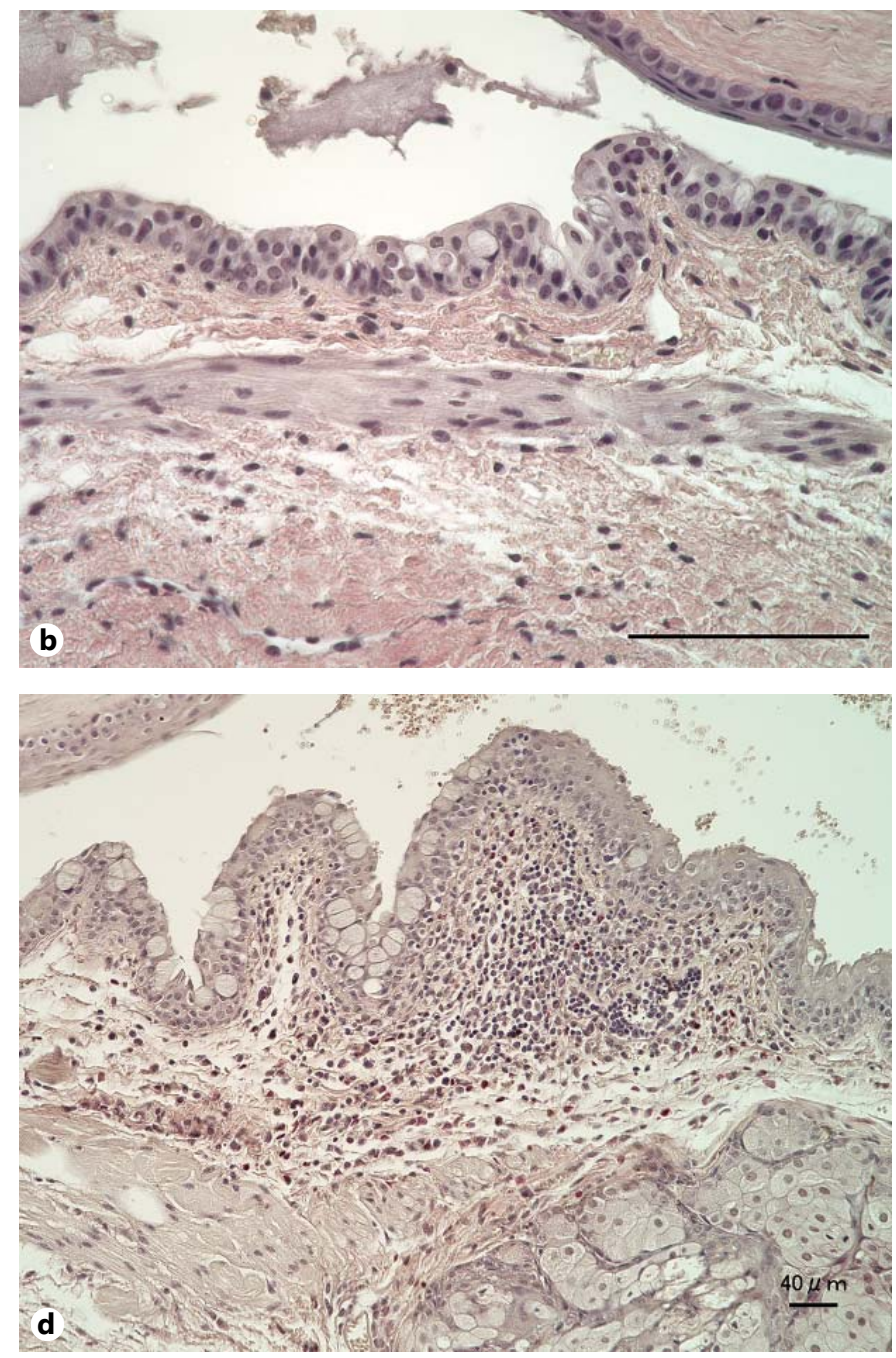

served. Extranodal lymphoid tissue-like structures with massive lymphocytic infiltration (iCALT), detected by DFS staining, were present in the subepithelial lesions of the palpebral conjunctiva in the AKC group (d), but were absent in the AD and C groups. Scale bar $=100 \mu \mathrm{m}(\mathbf{a}-\mathbf{c}), 40 \mu \mathrm{m}(\mathbf{d})$.

plasia region in the palpebral conjunctiva of sections from the AKC group. In the massive CD20-positive lymphocyte accumulation in the AKC mouse conjunctiva, CD4-positive T cells surrounded the CD20-positive lymphocyte aggregates (fig. 5).

\section{PCR Shows Elevated Expression of Th2 Chemokine $m R N A$ in AKC Mouse Conjunctiva}

To evaluate the allergic inflammation in the conjunctiva, the mRNA expression levels of Th2 chemokines and corresponding receptors (Ccl17, Ccl22 and Ccr4) in the conjunctival tissues was compared among the AKC-, 

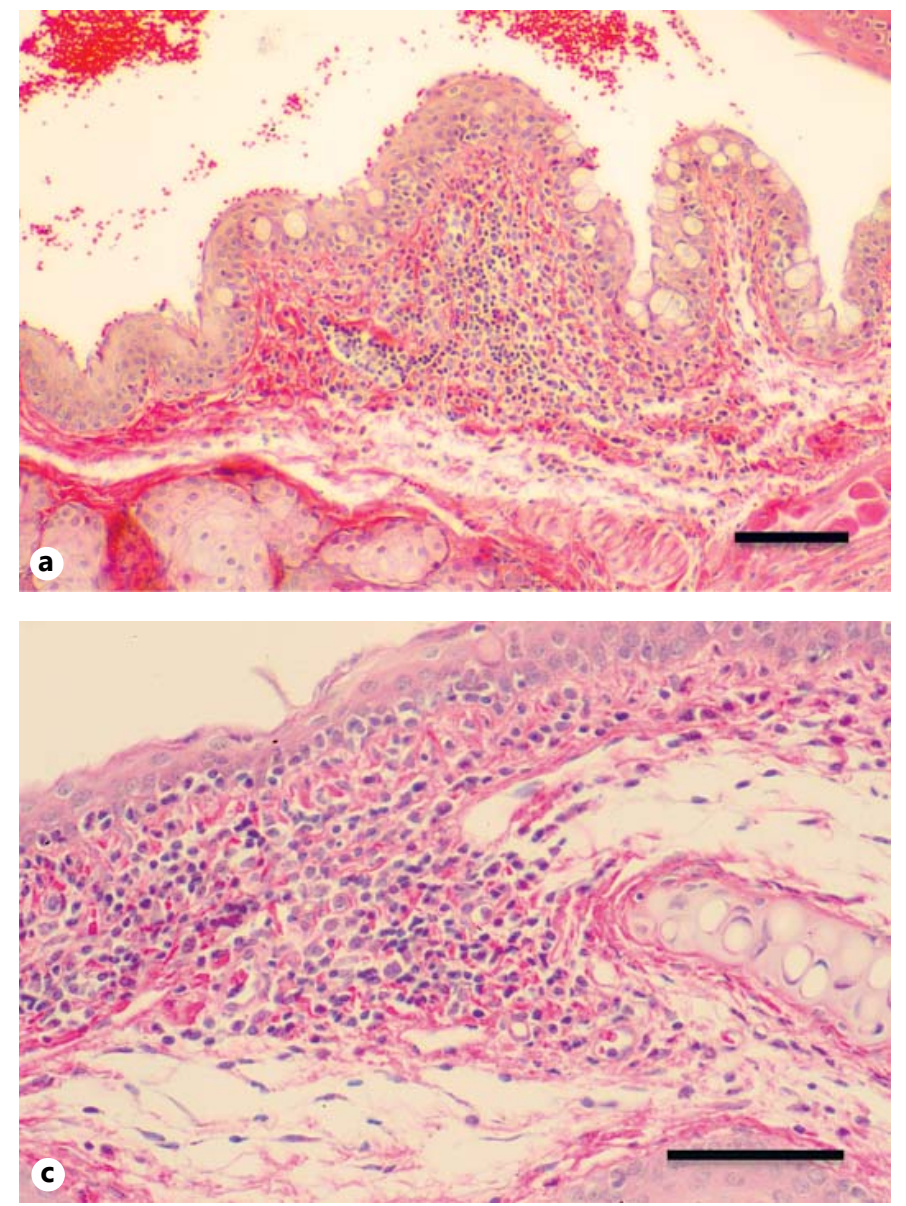

Fig. 4. Histological characteristics of iCALT and CALT in conjunctiva. Massive infiltration of inflammatory cells (iCALT), including CD20-positive lymphocyte infiltration in the central area, was observed in the subepithelial tissue of the palpebral conjunctiva in the AKC group by HE staining (a) and immunohistochemical meth-

Fig. 5. Immunohistochemistry of iCALT by immunofluorescent double-staining for CD4 and CD20. Frozen sections from AKC mice were probed with antibodies to CD4 and CD20. CD20-positive cells (Alexa Fluor ${ }^{\circledR}$ 647; pink) can be seen in the central area of iCALT in palpebral conjunctiva. CD4-positive cells (Alexa Fluor ${ }^{\circledR} 488$; green) can be seen in the periphery of the iCALT. Scale bar $=40 \mu \mathrm{m}$.
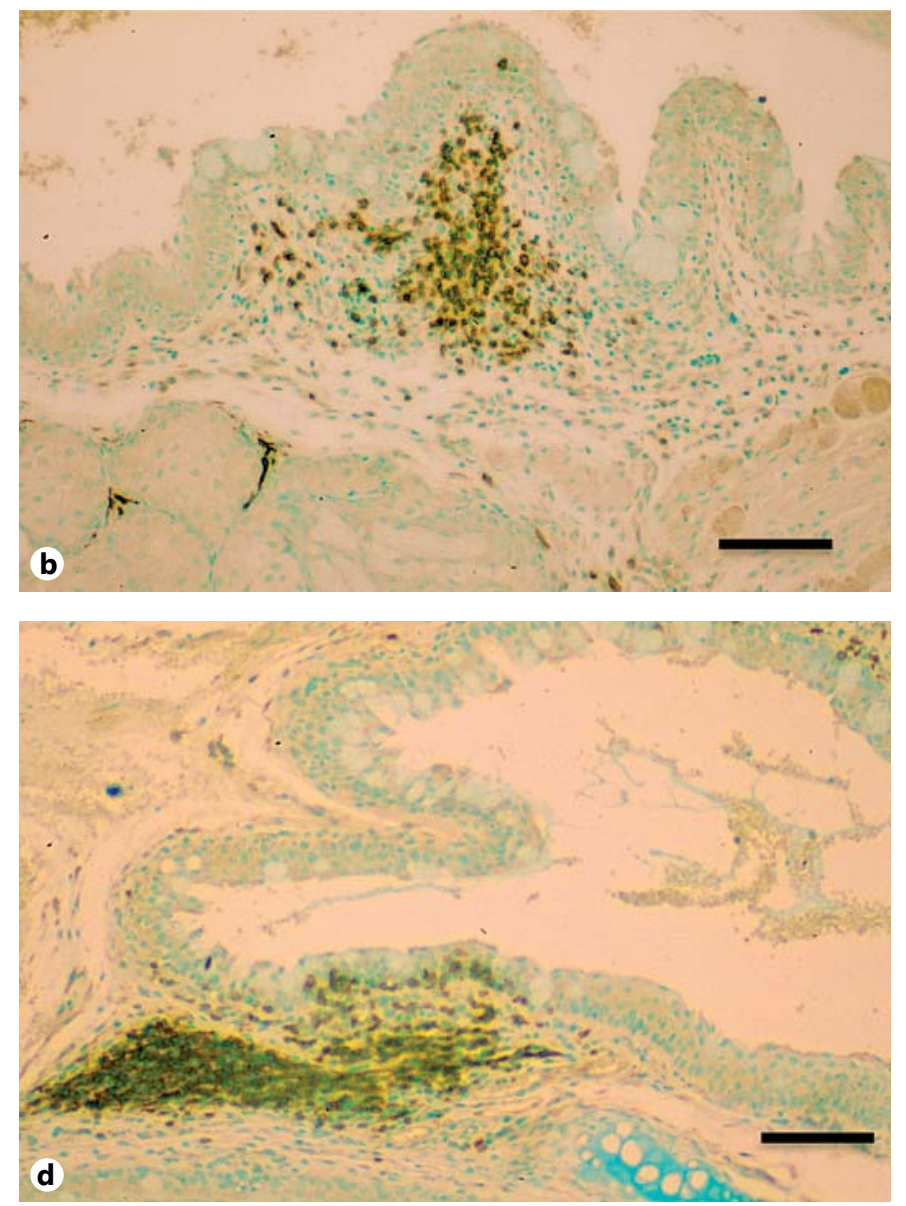

ods for CD20 (b). Massive infiltration of lymphocytes (CALT), including CD20-positive cell-infiltration in the central area, was also seen in the subepithelial tissue of the nictitating membrane in the $\mathrm{C}$ group by $\mathrm{HE}$ staining (c) and immunohistochemistry for CD20 (d). Scale bar $=100 \mu \mathrm{m}$.

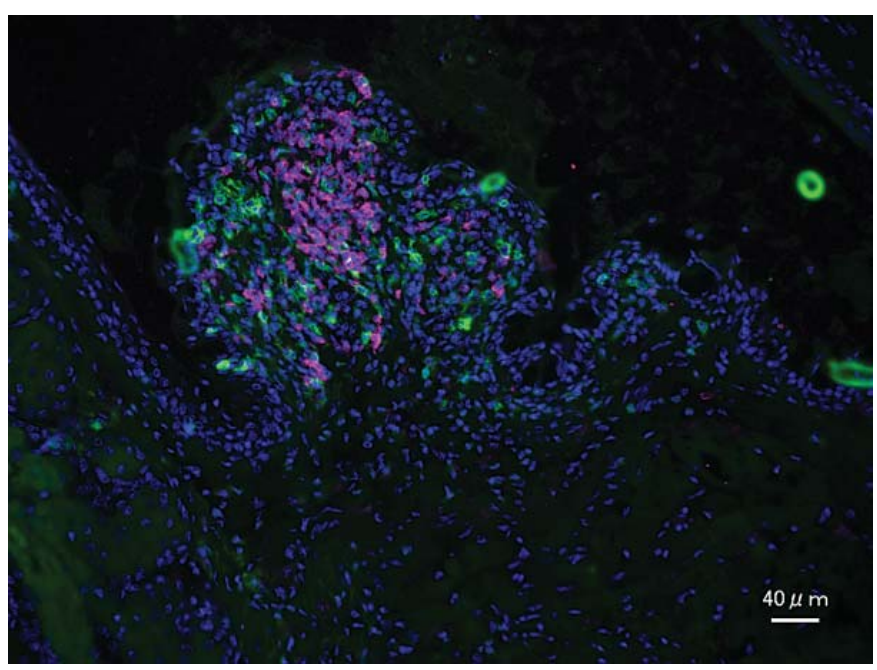




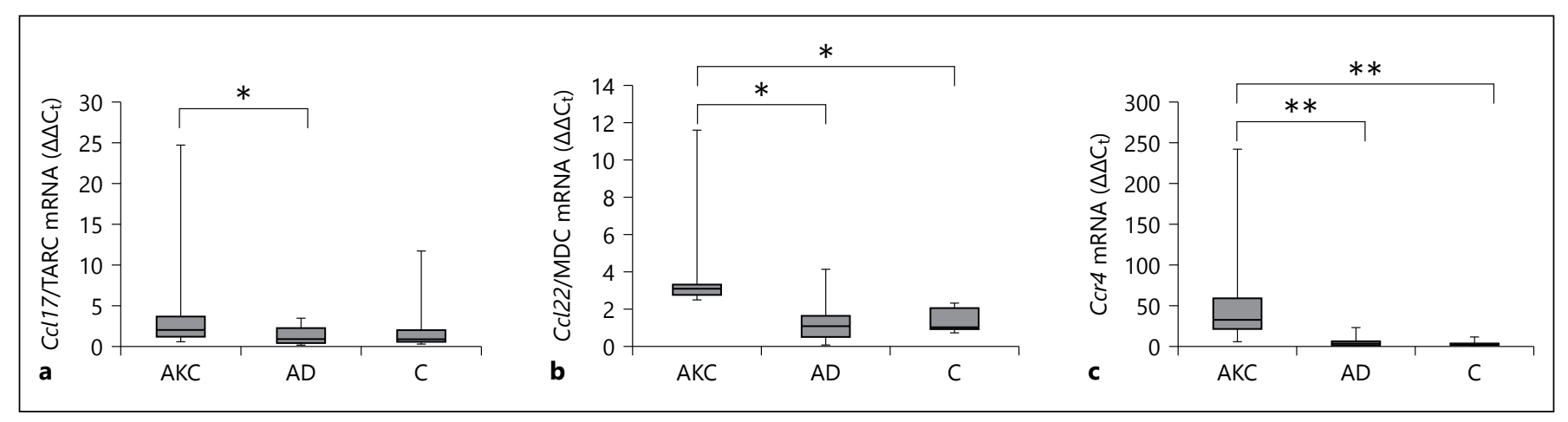

Fig. 6. Comparative study of mRNA expression levels of Th2-associated chemokines and their receptors in conjunctival tissues among the AKC, AD and C groups. Ccl17 (a), Ccl22 (b) and Ccr4 (c) messenger RNA (mRNA) expression in conjunctival tissues in the AKC group was significantly higher than those in the AD group. ${ }^{*} \mathrm{p}<$ $0.05,{ }^{* *} \mathrm{p}<0.01$, Steel test.

$\mathrm{AD}$ - and C-conjunctiva subgroups by real-time RTPCR. Ccl17 mRNA expression levels in the AKC-conjunctiva subgroup was significantly higher than that in the AD-conjunctiva subgroup (fig. 6a). Ccl22 and Ccr4 mRNA expression levels in the AKC-conjunctiva subgroup was significantly higher than that in the $\mathrm{AD}$ - and C-conjunctiva subgroups (fig. 6b, c).

\section{PCR Array and PCR Demonstrate High Levels of Chemokine mRNAs and T Cell Subclass-Related Transcriptional Factor mRNAs in iCALT}

To evaluate the iCALT-related factors that developed characteristically in iCALT, we compared the specimens obtained from the AKC-iCALT subgroup and those from the AKC-conjunctiva subgroup using various techniques.

PCR Array. In order to evaluate iCALT-related chemokines comprehensively, chemokine mRNA expression in specimens of the AKC-iCALT subgroup, obtained by LAMD, was compared with those of the AKC-conjunctiva subgroup, using PCR array. Chemokines were divided into three categories, depending on the n-fold increase in mRNA expression in the AKC-iCALT subgroup, as compared with the AKC-conjunctiva subgroup: (1) markedly increased (>10-fold); (2) moderately increased (5- to 9.9-fold); (3) not significantly increased or even decreased expression. A list of chemokines with marked or moderate increases is displayed in table 1. In terms of combinations of chemokines and their receptors, expression of the genes encoding the CCL20/CCR6, CCL17/CCR4 and CCL5/CCR3 axes markedly or moderately increased in the AKC-iCALT subgroup (table 1).

Real-Time PCR. The expression levels of chemokine genes (Ccl5, Ccl17, Ccl20, Cxcl3, Cxcl13 and Ccr7) in the
AKC-iCALT subgroup were quantitatively compared with those in the AKC-conjunctiva subgroup by real-time RT-PCR. Similar to the PCR array results, Ccl5, Ccl17, Ccl20, Cxcl3 and Ccr7 mRNA expression levels in the AKC-iCALT subgroup was significantly higher than those in the AKC-conjunctiva subgroup (fig. 7a-d, f). However, the Cxcl13 mRNA expression level in the AKC-iCALT subgroup was increased nonsignificantly (fig. 7e). Furthermore, expression of genes encoding $T$ cell subclassrelated transcription factors, such as T-bet, Gata-3, Foxp3 and ROR $\gamma \mathrm{t}$, was compared between the AKC-iCALT and AKC-conjunctiva subgroups. All these genes were expressed in both subgroups; however, Foxp 3 and T-bet levels in the AKC-iCALT subgroup were significantly higher than those in AKC-conjunctiva subgroup (table 2).

\section{Discussion}

In this study, we histopathologically confirmed the successful development of iCALT in the palpebral conjunctiva of the AKC mouse model. Comparison of AKC and $\mathrm{AD}$ mice revealed that the critical factor involved in the development of iCALT was severe allergic inflammation caused by percutaneous sensitization and ophthalmic challenge with the crude HDM antigen. According to the dual-allergen exposure hypothesis, per-eczematous sensitization as well as certain factors, including skin barrier damage, protease antigens and alarmin, skews immune responses toward Th2 reactions [24,25]. These immune responses may exacerbate the pathology of allergic diseases, such as AKC. We previously reported that the AKC mouse exhibits strong eosinophilic inflammation in 
Fig. 7. Comparison of chemokine mRNA expression between the AKC-iCALT and AKC-conjunctiva subgroups. In specimens of the AKC-iCALT and AKC-conjunctiva subgroups, the mRNA expression of $\mathrm{Ccl5} /$ RANTES (a), Ccl17/TARC (b), Ccl20/ $\operatorname{MIP}-3 a(\mathbf{c}), C x c l 3$ (d) and $C c r 7$ (f) in the AKC-iCALT subgroup was significantly higher than that in the AKC-conjunctiva subgroup. The mRNA expression of $\mathrm{Cxcl13}$ (e) in the AKC-iCALT subgroup was not statistically different from that in the AKCconjunctiva subgroup. ${ }^{*} \mathrm{p}<0.05,{ }^{* *} \mathrm{p}<$ 0.01 , Mann-Whitney U test.

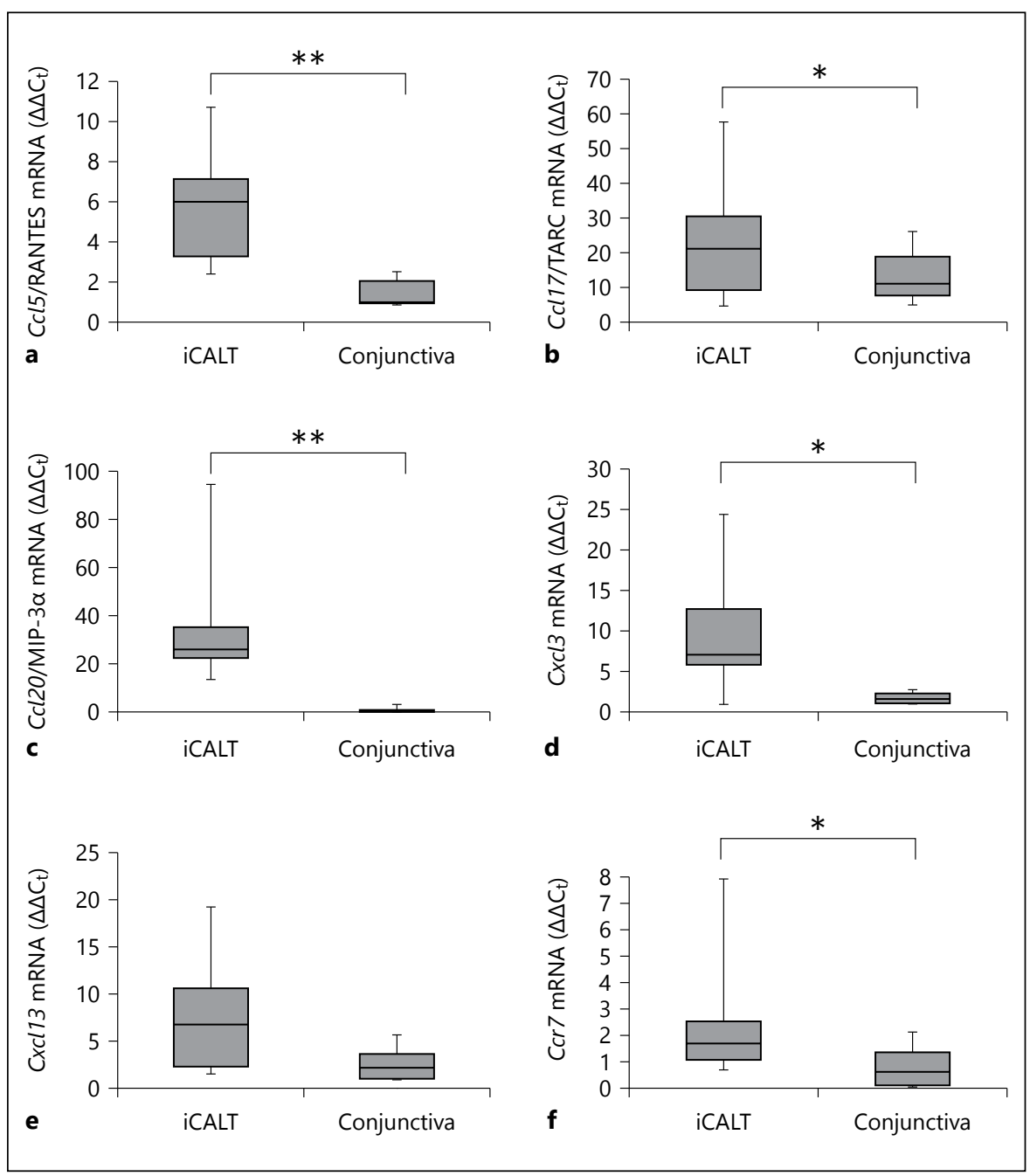

Table 1. PCR array analysis of the $\mathrm{n}$-fold increase in AKC-iCALT tissue compared with AKC-conjunctiva tissue

\begin{tabular}{llll}
\hline$>$ 10-fold increase & \multicolumn{2}{l}{ 5- to 9.9-fold increase } \\
\hline Ccl17 & 46.2 & Ccr5 & 9.8 \\
Ccr3 & 28.4 & Cxcr3 & 7.3 \\
Ccr6 & 27.5 & Cxcl15 & 6.9 \\
Cxcl10 & 22.3 & Cxcl1 & 6.5 \\
Ppbp & 19.4 & Ccr2 & 6.1 \\
Ccl20 & 18.9 & Cxcl5 & 5.7 \\
Ccl5 & 16.9 & Ccl9 & 5.5 \\
Tnf & 16.3 & & \\
Cxcl3 & 16.2 & & \\
Ccr7 & 13.6 & & \\
Ccr4 & 11.7 & & \\
\hline
\end{tabular}

$\mathrm{n}$-fold change $=$ value of mRNA in AKC-iCALT/value of mRNA in AKC-conjunctiva.
Table 2. $T$ cell subclass-related transcription factor expression in the AKC-iCALT and AKC-conjunctiva subgroups, real-time PCR $\left(\Delta \Delta \mathrm{C}_{\mathrm{t}}\right)$

\begin{tabular}{llll}
\hline & $\begin{array}{l}\text { AKC-iCALT } \\
\text { subgroup }\end{array}$ & $\begin{array}{l}\text { AKC-conjunctiva } \\
\text { subgroup }\end{array}$ & $\mathrm{p}^{1}$ \\
\hline T-bet & $2.2(0.9-7.2)$ & $1.0(0.6-1.1)$ & $<0.05$ \\
Gata-3 & $3.8(2.2-4.6)$ & $2.6(1.0-4.9)$ & n.s. \\
ROR $\gamma \mathrm{t}$ & $1.7(1.2-3.9)$ & $1.9(1.0-5.0)$ & n.s. \\
Foxp3 & $24.6(43.4-11.6)$ & $2.4(17.1-0.7)$ & $<0.01$ \\
\hline
\end{tabular}

Median values are presented with the range in parentheses. ${ }^{1}$ Mann-Whitney U test. 
the conjunctiva and HDM-specific IgE antibody level elevation in serum after percutaneous sensitization and ophthalmic challenge with the crude HDM antigen [21]. Furthermore, we here confirmed that the mRNA expression of Th2-associated chemokines, such as CCL17/ TARC and CCL22/MDC, is significantly increased in the conjunctiva of AKC mice, which suggested that marked conjunctival allergic inflammation was crucial to the development of iCALT.

The presence of CALT in conjunctival tissues has so far been reported in humans [12], chickens [13], rabbits [14], guinea pigs [15] and mice [16, 26, 27], although there are differences in the anatomical features, such as the location and size, across species. Histological characteristics common in CALT are the presence of lymphoepithelium, including $M$ cells [28], dome and parafollicular areas, including CD4-positive T cells, and a follicular area formed by $\mathrm{B}$ cells. In murine conjunctival tissues, the lymphoid tissues of the nictitating membrane are histologically similar to CALT $[16,26]$. Therefore, ectopic formation of secondary lymphoid follicles observed in the palpebral conjunctiva of AKC mice had histological characteristics of CALT, and were thus determined to be iCALT.

Furthermore, we identified iCALT-related chemokine analysis of LAMD specimens using PCR array and realtime RT-PCR. Comparative analysis between the AKCiCALT and AKC-conjunctiva subgroups using LAMD facilitates investigation of iCALT-related chemokines, as this method can exclude individual differences and an allergic inflammatory background. The CCL5/CCR3, CCL17/CCR4 and CCL20/CCR6 axes were significantly increased in the AKC-iCALT subgroup, as compared with the AKC-conjunctiva subgroup, and may represent crucial iCALT-related chemokines.

CCL20 is a chemokine that specifically binds CCR6expressing inflammatory cells, such as dendritic, Langerhans, B, memory T and IL-17-secreting CD4-positive T (Th17) cells. CCL20 is associated with the migration of Langerhans cells [29], B cells [30, 31], Th17 [32, 33] and type 9 helper T (Th9) cells [34] to inflammatory sites. However, the pathophysiological effects of CCL20 in severe ACD patients remain unclear. Additionally, CCL20 production by the lymphoepithelium-covering gut-associated lymphoid tissue (GALT), including Peyer's patches, has attracted attention; moreover, CCL20 and CXCL13 produced by the lymphoepithelium control the migration of myeloid dendritic cells to the dome area of the GALT, and likely play a crucial rule in GALT development in the gut [35]. CCL20 also contributes to the recruitment of CCR6-expressing B cells to the GALT [36]. Therefore, in-

Secondary Lymphoid Follicles in AKC

Mice creases in Ccl20 and Ccr6 mRNA in the iCALT of AKC mice may be pathologically similar to GALT development in the gut. In contrast, it has been reported that IL17-producing cells are important in the development of iBALT in a bronchial asthma mouse model [37]. Th17 cells are thought to produce CXCL13/BLC, chemokines inducing follicular formation comprised of B cells [38]. However, here, although Cxcl13 levels tended to increase, this increase was not significant in the AKC-iCALT subgroup as compared with the AKC-conjunctiva subgroup; thus, Cxcl13 may not be a crucial biomarker for iCALT formation in the conjunctiva.

Besides CCL20, mRNA levels of genes encoding CCL17/TARC and CCL5/RANTES were elevated in the AKC-iCALT subgroup as compared with the AKC-conjunctiva subgroup. The CCL17/CCR4 axis is associated with the migration of Th2 cells [39]. CCL17/TARC is also a serum biomarker for the severity of AD [40]. Additionally, vast amounts of CCL17/TARC are produced by the respiratory epithelium of asthmatic patients and exacerbate allergic inflammation in the respiratory track by promoting the migration of Th2 cells and eosinophils into the airway [41]. Therefore, higher Ccl17 expression in massive B cell lymphoid hyperplasic tissue, here termed iCALT, indicated that iCALT development might be involved in pathologic exacerbation of AKC.

Alternatively, the CCR3/RANTES axis is associated with the migration of Th1 cells and eosinophils [42]. RANTES is selectively expressed by germinal center (GC) $B$ cells in lymphoid tissues, and T cell homing within GCs is required for GC B cell-mediated IL-8 and RANTES chemoattraction [43]. Therefore, the CCR3/RANTES axis is thought to be involved in the formation of iCALT.

Furthermore, we found that T-bet and Foxp 3 expression in the AKC-iCALT subgroup was significantly increased as compared with that of the AKC-conjunctiva subgroup. T-bet, which is a Th1-specific T-box 1 transcription factor in mouse, is thought to be involved in the differentiation of Th1 cells and the class switch of B cells [44-46]. Th1 cell function is mainly associated with cellular immunity. T-bet is expressed in type 1 innate lymphoid cells (ILC1) [47] and a subset of B cell lymphoproliferative disorders $[48,49]$. Foxp 3 is a member of the FOX protein family and appears to function as a master regulator (transcription factor) in the development and function of regulatory T cells [50]. In contrast, in Peyer's patches, regulatory T cells (Foxp3+CD4+ T cells) differentiate into follicular helper $\mathrm{T}$ cells and are involved in IgA production [51]. Therefore, in future, analysis of Tbet- and Foxp3-positive cells will be important for eluci- 
dating the clinical significance of iCALT in ACD. We did not investigate the relationship between the subclass of $T$ and $\mathrm{B}$ cells and T-bet- or Foxp3-expressing cells; therefore, further studies are necessary.

The conjunctival histopathological characteristics of the AKC mouse indicated that both the development of iCALT by B cells, T-bet-positive lymphocytes and Foxp3positive lymphocytes, and allergic inflammation due to the infiltration of eosinophils and Th2, are crucial pathophysiological factors involved in AKC. Combined therapy with anti-IgE antibody (omalizumab) and anti-CD20 antibody (rituximab) has been effective in the treatment of severe $\mathrm{AD}$ [52]. Accordingly, in terms of treatment of severe AKC, it will be necessary to investigate the treatment of ectopic secondary lymphoid follicles, such as iCALT, in addition to antiallergic therapy. Lastly, iCALT- related chemokines can also potentially be used as critical biomarkers of localized, severe AKC by testing tear fluid in a clinical setting.

\section{Acknowledgements}

We wish to thank Miho Iduhara, $\mathrm{PhD}$, of Biostir Inc. for the preparation of the D. farinae body ophthalmic ointment. The authors thank Prof. Mitsuko Yuzawa (Division of Ophthalmology, Department of Visual Sciences, Nihon University School of Medicine) for editing this article.

\section{Disclosure Statement}

No author has any financial interest or conflict of interest to disclose.

\section{References}

1 Shoji J, Inada N, Sawa M: Antibody arraygenerated cytokine profiles of tears of patients with vernal keratoconjunctivitis or giant papillary conjunctivitis. Jpn J Ophthalmol 2006; 50:195-204.

2 Fukagawa K, Nakajima T, Tsubota K, Shimmura S, Saito H, Hirai K: Presence of eotaxin in tears of patients with atopic keratoconjunctivitis with severe corneal damage. J Allergy Clin Immunol 1999;103:1220-1221.

3 Shoji J, Kawaguchi A, Gotoh A, Inada N, Sawa M: Concentration of soluble interleukin-6 receptors in tears of allergic conjunctival disease. Jpn J Ophthalmol 2007;51:332-337.

4 Shoji J, Inada N, Sawa M: Evaluation of eotaxin-1, -2 , and -3 protein production and messenger RNA expression in patients with vernal keratoconjunctivitis. Jpn J Ophthalmol 2009;53:92-99.

5 Leonardi A, Curnow SJ, Zhan H, Calder VL: Multiple cytokines in human tear specimens in seasonal and chronic allergic eye disease and in conjunctival fibroblast culture. Clin Exp Allergy 2006;36:777-784.

6 Leonardi A: Allergy and allergic mediators in tears. Exp Eye Res 2013;117:106-117.

7 Botelho FM, Rangel-Moreno J, Fritz D, Randall TD, Xing Z, Richards CD: Pulmonary expression of oncostatin $\mathrm{M}$ (OCM) promotes inducible BALT formation independently of IL-6, despite a rule for IL-6 in OSM-driven pulmonary inflammation. J Immunol 2013; 191:1453-1464.

8 Hiramatsu K, Azuma A, Kudoh S, Desaki M, Takizawa H, Sugawara I: Inhalation of diesel exhaust for three months affects major cytokine expression and induces bronchusassociated lymphoid tissue formation in murine lungs. Exp Lung Res 2003;29:607622
9 Moyron-Quiroz JE, Rangel-Moreno J, Kusser K, Hartson L, Sprague F, Goodrich S, Woodland DL, Lund FE, Randall TD: Role of inducible bronchus-associated lymphoid tissue (iBALT) in respiratory immunity. Nat Med 2004;10:927-934.

10 Abu el-Asrar AM, van den Oord JJ, Geboes K, Missotten L, Emarah MH, Desmet V: Immunopathological study of vernal keratoconjunctivitis. Graefes Arch Clin Exp Ophthalmol 1989;227:374-379.

11 Matsuda A, Ebihara N, Yokoi N, Maruyama K, Hamuro J, Kinoshita S, Murakami A: Lymphoid neogenesis in the giant papillae of patients with chronic allergic conjunctivitis. J Allergy Clin Immunol 2010;126:1310-1312.

12 Knop N, Knop E: Conjunctiva-associated lymphoid tissue in the human eye. Invest Ophthalmol Vis Sci 2000;41:1270-1279.

13 Fix AS, Arp LH: Morphologic characterization of conjunctiva-associated lymphoid tissue in chickens. Am J Vet Res 1991;52:1852-1859.

14 Chandler JW, Axelrod AJ: Conjunctival-associated lymphoid tissue: a probable component of the mucosa-associated lymphoid system; in O'Connor GR (ed): Immunologic Diseases of the Mucous Membranes: Pathology, Diagnosis and Treatment. New York, Masson, 1980, pp 63-70.

15 Shoji J, Inada N, Saito K, Takaura N, Iwasaki $\mathrm{Y}$, Sawa M: Immunohistochemical study on follicular dendritic cell of conjunctiva-associated lymphoid tissue. Jpn J Ophthalmol 1998; $42: 1-7$

16 Sakimoto T, Shoji J, Inada N, Saito K, Iwasaki Y, Sawa M: Histological study of conjunctivaassociated lymphoid tissue in mouse. Jpn J Ophthalmol 2002;46:364-369.

17 Tomasi TB Jr, Larson L, Challacombe S, McNabb P: Mucosal immunity: the origin and migration patterns of cells in the secretory system. J Allergy Clin Immunol 1980;65: 12-19.

18 Iwaya Y, Kobayashi M, Momose M, Hiraoka $\mathrm{N}$, Sakai Y, Akamatsu T, Tanaka E, Ohtani H, Fukuda M, Nakayama J: High levels of $\mathrm{FOXP}^{+}$regulatory $\mathrm{T}$ cells in gastric MALT lymphoma predict responsiveness to Helicobacter pylori eradication. Helicobacter 2013; 18:356-362.

19 Heishi M, Imai Y, Katayama H, Hashida R, Ito M, Shinagawa A, Sugita Y: Gene expression analysis of atopic dermatitis-like skin lesions induced in $\mathrm{NC} / \mathrm{Nga}$ mice by mite antigen stimulation under specific pathogen-free conditions. Int Arch Allergy Immunol 2003; 132:355-363.

20 Yamamoto M, Haruna T, Yasui K, Takahashi H, Iduhara M, Takaki S, Deguchi M, Arimura A: A novel atopic dermatitis model induced by topical application with Dermatophagoides farinae extract in NC/Nga mice. Allergol Int 2007;56:139-148.

21 Hara Y, Shoji J, Hori S, Ishimori A, Kato H, Inada N, Sawa M: Evaluation of eosinophilic infiltration in a novel murine atopic keratoconjunctivitis model induced by crude Dermatophagoides farinae antigen. Allergol Int 2012;61:331-339.

22 Foster CS, Rice BA, Dutt JE: Immunopathology of atopic keratoconjunctivitis. Ophthalmology 1991;98:1190-1196.

23 Nakanishi Y, Mizutani G, Sano M, Oinuma T, Nemoto N: Comparison of HER2 mRNA amplification with immunohistochemistry in human breast cancer using laser assisted microdissection technique. Acta Histochem Cytochem 2004;37:73-79.

24 Lack G: Update on risk factors for food allergy. J Allergy Clin Immunol 2012;129:1187-1197. 
25 Matsumoto K, Saito H: Epicutaneous immunity and onset of allergic disease - per'eczema'tous sensitization drives the allergy march. Allergol Int 2013;62:291-296.

26 Steven P, Rupp J, Hüttmann G, Koop N, Lensing $\mathrm{C}$, Laqua $\mathrm{H}$, Gebert A: Experimental induction and three-dimensional two-photon imaging of conjunctiva-associated lymphoid tissue. Invest Ophthalmol Vis Sci 2008;49:1512-1517.

27 Siebelmann S, Gehlsen U, Hüttmann G, Koop N, Bölke T, Gebert A, Stern ME, Niederkorn JY, Steven P: Development, alteration and real time dynamics of conjunctiva-associated lymphoid tissue. PLoS One 2013;8:e82355.

28 Giuliano EA, Moore CP, Phillips TE: Morphological evidence of $M$ cells in healthy canine conjunctiva-associated lymphoid tissue. Graefes Arch Clin Exp Ophthalmol 2002;240: 220-226.

29 Charbonnier AS, Kohrgruber N, Kriehuber E, Stingl G, Rot A, Maurer D: Macrophage inflammatory protein $3 a$ is involved in the constitutive trafficking of epidermal Langerhans cells. J Exp Med 1999;190:1755-1768.

30 Krzysiek R, Lefevre EA, Bernard J, Foussat A, Galanaud P, Louache F, Richard Y: Regulation of CCR6 chemokine receptor expression and responsiveness to macrophage inflammatory protein-3a/CCL20 in human B-cells. Blood 2000;96:2338-2345.

31 Liao F, Shirakawa AK, Foley JF, Rabin RL, Farber JM: Human B-cells become highly responsive to macrophage-inflammatory protein-3 $\alpha / C C$ chemokine ligand-20 after cellular activation without changes in CCR6 expression or ligand binding. J Immunol 2002;168:4871-4880.

32 Dohlman TH, Chauhan SK, Kodati S, Hua J, Chen Y, Omoto M, Sadrai Z, Dana R: The CCR6/CCL20 axis mediates Th17 cell migration to the ocular surface in dry eye disease. Invest Ophthalmol Vis Sci 2013;54:4081-4091.

33 Hirota K, Yoshitomi H, Hashimoto M, Maeda S, Teradaira S, Sugimoto N, Yamaguchi T, Nomura T, Ito H, Nakamura T, Sakaguchi N, Sakaguchi S: Preferential recruitment of CCR6expressing Th17 cells to inflamed joints via CCL20 in rheumatoid arthritis and its animal model. J Exp Med 2007;204:2803-2812.

34 Kara EE, Comerford I, Bastow CR, Fenix KA, Litchfield W, Handel TM, McColl SR: Distinct chemokine receptor axes regulate Th9 cell trafficking to allergic and autoimmune inflammatory sites. J Immunol 2013;191:11101117.

35 Hase K, Murakami T, Takatsu H, Shimaoka T, Iimura M, Hamura K, Kawano K, Ohshima $\mathrm{S}$, Chihara R, Itoh $\mathrm{K}$, Yonehara S, Ohno $\mathrm{H}$ : The membrane-bound chemokine CXCL16 expressed on follicle-associated epithelium and $\mathrm{M}$ cells mediates lympho-epithelial interaction in GALT. J Immunol 2006;176:43-51.

36 Williams IR: CCR6 and CCL20: partners in intestinal immunity and lymphorganogenesis. Ann N Y Acad Sci 2006;1072:52-61.

37 Rangel-Moreno J, Carragher DM, de la Luz Garcia-Hernandez M, Hwang JY, Kusser K, Hartson L, Kolls JK, Khader SA, Randall TD: The development of inducible bronchus-associated lymphoid tissue depends on IL-17. Nat Immunol 2011;12:639-646.

38 Takagi R, Higashi T, Hashimoto K, Nakano K, Mizuno Y, Okazaki Y, Matsushita S: B cell chemoattractant CXCL13 is preferentially expressed by human Th17 cell clones. J Immunol 2008; 181:186-189.

39 Vestergaard C, Deleuran M, Gesser B, Larsen CG: Thymus- and activation-regulated chemokine (TARC/CCL17) induces a Th2-dominated inflammatory reaction on intradermal injection in mice. Exp Dermatol 2004;13:265271.

40 Jahnz-Rozyk K, Targowski T, Paluchowska E, Owczarek W, Kucharczyk A: Serum thymus and activation-regulated chemokine, macrophage-derived chemokine and eotaxin as markers of severity of atopic dermatitis. Allergy 2005;60:685-688.

41 Ying S, O’Connor B, Ratoff J, Meng Q, Fang C, Cousins D, Zhang G, Gu S, Gao Z, Shamji B, Edwards MJ, Lee TH, Corrigan CJ: Expression and cellular provenance of thymic stromal lymphopoietin and chemokines in patients with severe asthma and chronic obstructive pulmonary disease. J Immunol 2008; 181:2790-2798.

42 Kaplan AP: Chemokines, chemokine receptors and allergy. Int Arch Allergy Immunol 2001;124:423-431.

43 Sims-Mourtada JC, Guzman-Rojas L, Rangel R, Nghiem DX, Ullrich SE, Garret C, Cain K, Martinez-Valdez H: In vivo expression of interleukin-8, and regulated on activation, normal, T-cell expressed, and secreted, by human germinal centre B lymphocytes. Immunology 2003;110:296-303.

44 Sabot SJ, Kim ST, Costa GL, Zhang X, Fathoming CG, Glitches LH: A novel transcription factor, T-bet, directs Th1 lineage commitment. Cell 2000;100:655-669.

45 Matsuyama M, Ishii Y, Yagita Y, Otsuka S, Anon S, Matsudo Y, Morishima Y, Yoho K, Takahashi S, Ogawa K, Hogaboam CM, Hizawa N: Role of Th1/Th17 balance regulated by T-bet in a mouse model of Mycobacterium avium complex disease. J Immunol 2014;192: 1707-1717.

46 Peng SL, Szabo SJ, Glimcher LH: T-bet regulates IgG class switching and pathogenic autoantibody production. Proc Natl Acad Sci U S A 2002;99:5545-5550.

47 Bernink JH, Peters CP, Munneke M, te Velde AA, Meijer SL, Weijer K, Hreggvidsdottir HS, Heinsbroek SE, Legrand N, Buskens CJ, Bemelman WA, Mjösberg JM, Spits H: Human type 1 innate lymphoid cells accumulate in inflamed mucosal tissue. Nat Immunol 2013;14: 221-229.

48 Dorfman DM, Hwang ES, Shahsafaei A, Glimcher LH: T-bet, a T-cell-associated transcription factor, is expressed in a subset of B-cell lymphoproliferative disorders. Am J Clin Pathol 2004;122:292-297.

49 Bob R, Falini B, Marafioti T, Paterson JC, Pileri S, Stein H: Nodal reactive and neoplastic proliferation of monocytoid and marginal zone B-cells: an immunoarchitectural and molecular study highlighting the relevance of IRTA 1 and T-bet as positive markers. Histopathology 2013;63:482-498.

50 Zhang L, Zhao Y: The regulation of Foxp3 expression in regulatory $\mathrm{CD} 4^{+} \mathrm{CD} 25^{+} \mathrm{T}$ cells: multiple pathways on the road. J Cell Physiol 2007;211:590-597.

51 Tsuji M, Komatsu N, Kawamoto S, Suzuki K, Kanagawa O, Honjo T, Hori S, Fagarasan S: Preferential generation of follicular B helper $\mathrm{T}$ cells from Foxp $3^{+} \mathrm{T}$ cells in gut Peyer's patches. Science 2009;323:1488-1492.

52 Sánchez-Ramón S, Eguíluz-Gracia I, Rodríguez-Mazariego ME, Paravisini A, ZubeldiaOrtuño JM, Gil-Herrera J, Fernández-Cruz E, Suárez-Fernández R: Sequential combined therapy with omalizumab and rituximab: a new approach to severe atopic dermatitis. J Investig Allergol Immunol 2013;23:190-196.
Secondary Lymphoid Follicles in AKC

Mice
Int Arch Allergy Immunol 2015;167:147-157 DOI: $10.1159 / 000437424$ 